\title{
EFECTO DE UN PROGRAMA DE HIDROQUINESIA Y ACONDICIONAMIENTO FÍSICO EN LA MOVILIDAD ARTICULAR DE ADULTAS MAYORES
}

\author{
Cinthya Campos Salazar y Luis Solano Mora \\ Escuela de Educación Física y Deportes \\ Universidad de Costa Rica, San José Costa Rica \\ E-mail: cinthyac@cariari.ucr.ac.cr
}

\begin{abstract}
Resumen
Campos, C. y Solano, L. (2001). Efecto de un programa de hidroquinesia y acondicionamiento físico en la movilidad articular de adultas mayores. Revista en Ciencias del Ejercicio y la Salud, 1(2), 12-18. El propósito de este estudio fue determinar el efecto de un programa combinado de hidroquinesia y acondicionamiento físico y otro de acondicionamiento físico solamente en la movilidad articular en las caderas de un grupo de adultas mayores. Sesenta y tres sujetos (edad promedio $=66.06$ años, S.D. \pm 6.77 ) fueron asignados a los grupos control, hidroterapia y acondicionamiento físico y solo acondicionamiento físico. La duración del programa fue de una hora por sesión, dos veces a la semana durante 16 semanas. Se aplicó un pretest y un postest de movilidad articular para medir la movilidad global de las caderas (flexión, abducción, aducción, rotación interna y externa). Las variables independientes fueron lado izquierdo y derecho vs. grupos de tratamiento. Los resultados indicaron lo siguiente: el grupo control no varío la movilidad articular en las mediciones de flexión, rotación interna y externa ( $p>0.05)$ mientras que para la mediciones de abducción y aducción disminuyó significativamente $(p<0.05)$. En cuanto a los grupos de tratamiento, el grupo que realizó el programa combinado de hidroquinesia y acondicionamiento físico aumentó significativamente $(p<0.05)$ la movilidad articular a nivel de flexión, abducción y aducción, en tanto el grupo que realizó el programa de acondicionamiento físico solamente, mejoró significativamente $(p<0.05)$ en flexión, abducción, aducción, rotación interna como externa. En conclusión se recomienda la práctica de actividad física tanto en el agua como fuera de ella, pues mejora los rangos de movilidad articular en cadera, a nivel global en personas adultas mayores. PALABRAS CLAVES: adulto mayor, movilidad articular.
\end{abstract}

\section{INTRODUCCIÓN}

El interés por estudiar tanto la respuesta como la adaptación al ejercicio en la población mayor 60 años, a crecido con el paso de los años, paralelo al aumento en la esperanza de vida que se experimenta prácticamente en todo el mundo.

El proceso de envejecimiento es reconocido como un proceso natural, universal, de pérdida de adaptación, el cual ocurre constantemente y es irreversible (Miller, 1994), sin embargo la producción científica brinda información consistente en relación con la capacidad de adaptación de los distintos sistemas corporales en la población mayor (Israel, 1992; Williams, 1999; Willmore y Costill, 1999).

Al respecto, se ha observado que ocurre "una adaptabilidad para el estímulo persistente de la fuerza" (Israel, 1992, p.324), aunque parece que el nivel de entrenabilidad de la misma, disminuye conforme pasa el tiempo.
Por su parte, Aniansson y Gustafson (1981) en su estudio, indicaron que un grupo de adultos mayores con un rango de edad que oscilaba entre los 69 y los 74 años, presentaron ganancia de fuerza muscular a un nivel muy similar que en un grupo de hombres jóvenes.

Otros autores (Clarkson y Kroll, 1978 y Spirduso y Clifford, 1978) han comprobado en sus estudios que los programas de ejercicios mejoran significativamente las capacidades físicas y motoras de los adultos mayores; resultados que han sido consistentes hasta la fecha (Rikli y Busch, 1986; Baylor y Spirduso, 1988; Merdith y otros, 1989; Fiatarone y otros, 1990; Harris y otros, 2001).

De acuerdo con Clark (1986), los adultos mayores que logran disminuir la incidencia de daños estructurales es porque en su mayoría acompañaron sus actividades tempranas con el deporte.

Una de las consecuencias de daños en las estructuras corporales, que se asocia con el 
envejecimiento, es la pérdida de movilidad articular, es decir, pérdida de la flexibilidad.

Carter y otros (1987) indicaron que la integridad funcional de una articulación va a estar influenciada por los grupos musculares asociados, por lo tanto, la musculatura fuerte, como los ligamentos tensos, redundan en cápsulas articulares estables.

Así mismo, Mcginnis (1992) manifestó que la actividad física puede mejorar la fortaleza músculo esquelética y la flexibilidad y así reducir riesgos a sufrir daños estructurales como funcionales.

Por su parte, Ortiz (1998) indicó que el ejercicio ayuda a prevenir ciertas alteraciones en la marcha y síndromes en el aparato osteolocomotor. El mismo autor $(1998$, p.16) afirmó, también, que "el valor preventivo del ejercicio en el adulto mayor, es lograr la promoción del ejercicio cotidiano previo a que ocurra una deficiencia por una lesión, para evitar así la minusvalez, con la consecuente limitación de las funciones normales".

Apoyando lo anterior, el American College of Sports Medicine (ACSM, por sus siglas en inglés) (2000, p.156), indica que la "función músculo esquelética óptima requiere que un adecuado rango de movimiento sea mantenido en todas las articulaciones"; siendo de vital importancia, sobretodo, en los adultos mayores, debido a que una pérdida de flexibilidad se relaciona con una disminución en su capacidad funcional física.

Según Nieman (2000), la pérdida de rango de movimiento articular provoca una rigidez de los tendones, músculos $\mathrm{y}$ otros tejidos que se encuentran alrededor de la articulación; lo cual indica la importancia de trabajar la movilidad articular, reiterado por Schroeder y otros (1998) y Klein (2001), al citar que la flexibilidad es un factor importante para mejorar la función física de los adultos mayores y como consecuencia la calidad de vida.

Diversos estudios (Shepard, 1994; Sergent, 1995; Lan y otros, 1998; Mobily y otros, 1998) han encontrado que la actividad física provoca incrementos significativos de la flexibilidad en adultos mayores. En su tesis Sergent (1995) encontró que un programa de ejercicios de estiramiento, de fuerza de intensidad baja y ejercicios aeróbicos a moderada intensidad, de 6 meses de duración, provoca mejorías significativas de la flexibilidad.

Por su parte Lan y otros (1998) hallaron que un programa de Tai Chi Chuan, con una duración de 11 meses y 5 veces a la semana, el cual consistió en 20 minutos de calentamiento, 24 minutos de Tai Chi Chuan y 10 minutos de estiramiento, incrementó significativamente el rango de movimiento articular.

Así mismo, Mobily y otros (1998) mostraron que un programa de 8 semanas de pesas progresivo logró mejorar aumentar significativamente la flexibilidad.

Solano y Vargas (1999), en su estudio, comparan el efecto de tres tipos de programas de actividad física en la movilidad articular de hombros y cadera, uno de los grupos asistió a un programa regular de natación, otro asistió a un programa de hidroquinesia o ejercicios en el agua, y un tercer grupo realizó un programa combinado de natación $\mathrm{y}$ de hidroquinesia. Los resultados indicaron que en la articulación de hombros, los porcentajes de cambio de los tres grupos que se ejercitaron fueron significativamente distintos que los del grupo control únicamente. En cuanto a las caderas los porcentajes de cambio de los grupos que realizaron hidroquinesia e hidroquinesia-natación fueron significativamente distintos del grupo que realizó natación solamente, y el grupo control. Estos resultados indican que en la movilidad articular de la cadera, la hidroquinesia per se, o al combinarla con la natación es la que marca la diferencia.

Por tanto, considerando la importancia de estimular la movilidad articular en la población mayor, según documentación presentada, y por los resultados obtenidos en las investigaciones descritas sobre el tema, se plantea la siguiente interrogante:

¿Se obtendrán resultados distintos al aplicar un programa combinado de hidroquinesia y ejercicios de acondicionamiento físico $\mathrm{y}$ un programa solamente de acondicionamiento físico en la movilidad articular de la cadera en población mayor de 60 años? 


\section{METODOLOGÍA}

\section{Sujetos}

En el estudio participaron 63 mujeres con edad promedio y desviación estándar de $66.1 \pm 6.8$ años, respectivamente y cuyo lado dominante era el lado derecho.

\section{Instrumentos}

Para el presente trabajo se contó con una camilla exploratoria, hojas de anotación de rangos de movilidad articular y un goniómetro manual con una precisión de \pm 1 grado. La prueba utilizada para medir rango de movilidad articular es la descrita por Heyward (1998). La unidad de medición fue en grados, lo cual, según la misma autora (1998) resulta de mayor funcionalidad que una medición lineal de la flexibilidad, puesto que el movimiento propio de las articulaciones, es de tipo angular. Para las mediciones de flexión, abducción, aducción la persona debía estar en posición supina, y para las mediciones de rotación interna, rotación externa la persona debía estar sentada (Heyward, 1998). La tabla 1 detalla el protocolo de la prueba.

\section{Procedimientos}

Una vez obtenida la aprobación por parte de los participantes, se realizaron las mediciones de movilidad articular en la cadera (flexión, abducción, aducción, rotación interna y rotación externa), la extensión de cadera no se midió pues al tener que hacer las mediciones sin calentamiento previo y como lo que se buscaba era alcanzar el rango máximo de movimiento, ello podría provocar compresión del nervio ciático y consecuentemente generar un cuadro de dolor.

Los rangos de movimiento se midieron tanto del lado derecho como del lado izquierdo, considerando que, aún cuando desde el punto de vista biológico se consideran ambos lados (derechaizquierda) simétricos, desde el punto de vista funcional se presenta asimetría (Rigal, 1987). El autor refuerza el concepto de la asimetría funcional al referirse a lado dominante y lado débil; por tal motivo se decidió medir los rangos de movimiento articular tanto en lado derecho como izquierdo y estudiarlo como una variable independiente. Se trabajó con tres grupos, dos experimentales y uno control. Los grupos eran intactos, por lo que una vez realizadas las mediciones se procedió a la aplicación de los tratamientos. El tratamiento aplicado a los grupos experimentales tuvo una duración de 16 semanas y una frecuencia de 2 veces por semana. El grupo control, durante el período de aplicación de los programas de ejercicio, se reunía una vez por semana a realizar manualidades.

\section{Tabla 1}

Descripción del Protocolo de Mediciones Universal con Goniómetro para rangos de Movilidad Articular

\begin{tabular}{lll}
\hline \multicolumn{1}{c}{ Eje Rotación } & \multicolumn{1}{c}{$\begin{array}{c}\text { Segmento } \\
\text { estático }\end{array}$} & Segmento móvil \\
\hline $\begin{array}{l}\text { Trocánter mayor } \\
\text { fémur (flexión) }\end{array}$ & $\begin{array}{l}\text { Eje longitudinal } \\
\text { del muslo en } \\
\text { decúbito dorsal }\end{array}$ & $\begin{array}{l}\text { Ídem estático } \\
\text { y acompaña el } \\
\text { muslo en la } \\
\text { flexión }\end{array}$ \\
$\begin{array}{l}\text { Espina Ilíaca, } \\
\text { antero-superior } \\
\text { (abducción- } \\
\text { aducción) }\end{array}$ & $\begin{array}{l}\text { Eje transversal } \\
\text { decúbito dorsal }\end{array}$ & $\begin{array}{l}\text { Ídem estático y } \\
\text { acompaña al } \\
\text { segmento; la } \\
\text { punta del pie } \\
\text { mantenerla hacia } \\
\text { arriba }\end{array}$ \\
$\begin{array}{l}\text { Parte media } \\
\text { rótula (rotación } \\
\text { in terna-externa) }\end{array}$ & $\begin{array}{l}\text { Eje longitudinal } \\
\text { de la pierna, } \\
\text { posición sentada }\end{array}$ & $\begin{array}{l}\text { Ídem estático y } \\
\text { acompaña la } \\
\text { pierna. }\end{array}$ \\
\hline
\end{tabular}

Los tratamientos aplicados fueron los siguientes:

1. Combinado (hidroquinesia y acondicionamiento físico): Los participantes de este grupo realizaron durante la semana una sesión de hidroquinesia, en la que practicaron movimientos específicos de movilidad para la cadera como el caminar, "jumping jacks", desplazamientos, entre otros, y una sesión de acondicionamiento físico, la cual contempló ejercicio de tipo aeróbico como ejercicio localizado.

2. El programa de solo acondicionamiento físico: consistió de dos sesiones por semana y contempló tanto los ejercicios como la distribución que en el programa combinado, ejercicio aeróbico y ejercicio localizado. Finalizadas 
las 16 semanas se realizó nuevamente el protocolo completo para la medición de los rangos de movilidad articular en los cinco movimientos de cadera.

\section{Análisis Estadístico}

Para realizar el análisis de los datos se utilizó un ANOVA de tres vías 3 × 2 × 2 (grupos $\mathrm{x}$ lateralidad $\mathrm{x}$ mediciones) mixta, $\mathrm{y}$ se calcularon los ectos simples y post-hoc de Tukey en los casos necesarios

\section{RESULTADOS}

La estadística descriptiva (promedio y desviación estándar) para cada medición de movilidad articular por grupo, se presenta en la tabla 2. El dato corresponde al promedio de las mediciones de ambos lados (izquierdo y derecho), debido a que no hubo diferencia significativa entre ellos, como se verá en los ANOVA más adelante.

Tabla 2

\section{Promedios y desviaciones estándar para cada medición en la flexibilidad de los cinco movimientos de la cadera}

\begin{tabular}{|c|c|c|c|c|}
\hline \multirow[t]{2}{*}{ Movimiento/medición } & \multicolumn{2}{|c|}{$\begin{array}{c}\text { Pretest } \\
\text { (grados) }\end{array}$} & \multicolumn{2}{|c|}{$\begin{array}{c}\text { Postest } \\
\text { (grados) }\end{array}$} \\
\hline & $\mathrm{X}$ & DS & $X$ & DS \\
\hline Flexión & 59.2 & \pm 16.5 & 58.3 & \pm 16.1 \\
\hline Combinado & 63.4 & \pm 13.4 & 69.5 & \pm 12.6 \\
\hline Acond. Físico & 62.9 & \pm 14.7 & 71.7 & \pm 12.9 \\
\hline Abducción & 37.1 & \pm 10.3 & 31.7 & \pm 8.3 \\
\hline Combinado & 26.9 & \pm 7.2 & 33.4 & \pm 8.4 \\
\hline Acond. Físico & 30.8 & \pm 8.7 & 34.8 & \pm 8.4 \\
\hline Aducción & 33.8 & \pm 11.1 & 28.8 & \pm 5.3 \\
\hline Combinado & 20.2 & \pm 5.1 & 26.8 & \pm 5.3 \\
\hline Acond. Físico & 22.9 & \pm 5.7 & 27.4 & \pm 5.7 \\
\hline Rotación & 28.8 & \pm 6.1 & 29.2 & \pm 6.7 \\
\hline Combinado & 29.1 & \pm 8.4 & 28.8 & \pm 7.7 \\
\hline Acond. Físico & 27.6 & \pm 7.3 & 30.3 & \pm 7.0 \\
\hline Rotación & 31.2 & \pm 8.0 & 29.2 & \pm 6.7 \\
\hline Externa & 24.8 & \pm 4.9 & 25.1 & \pm 4.4 \\
\hline Acond. Físico & 22.6 & \pm 4.8 & 25.7 & \pm 4.5 \\
\hline
\end{tabular}

Con base en el análisis estadístico ANOVA de tres vías (grupos $\mathrm{x}$ lateralidad $\mathrm{x}$ mediciones) mixta para los cinco rangos de movimiento articular en estudio, flexión, abducción, aducción y rotación interna y externa de caderas, hubo interacción doble significativa.

Analizando los efectos simples de las interacciones dobles, para la medición de flexión de cadera el grupo control no varió su movilidad articular entre el pretest y el postest ( $\mathrm{p}>0.05)$; por el contrario, tanto el grupo que realizó el programa combinado de hidroquinesia y acondicionamiento físico como el grupo que sólo realizó ejercicios de acondicionamiento físico (ver figura 1), sí manifestaron cambios significativos entre el pretest y el postest, igualmente en lado derecho como en lado izquierdo $(\mathrm{p}<0.05)$.

Para las mediciones de abducción y aducción, los efectos simples mostraron que en todos los grupos hubo diferencia significativa $(p<0.05)$ entre el pretest y el postest; el grupo control disminuyó mientras que los grupos que se ejercitaron incrementaron la movilidad articular en los movimientos de abducción y aducción de caderas igualmente en lado izquierdo y lado derecho, (ver figura 2 y 3 ).

Figura 1

Promedios de mediciones Inicial y Final en Flexión de cadera para grupos de Ejercicio y el grupo control

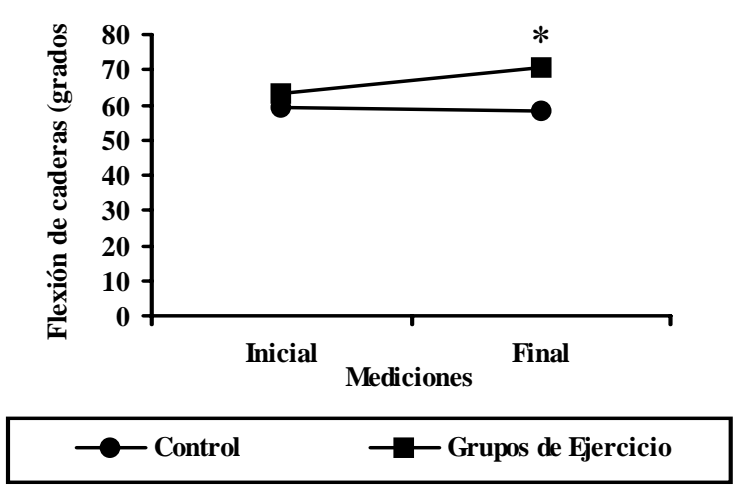

* p $<0.05$ Diferencia significativa entre la medición inicial y final en los dos grupos que realizaron ejercicio 
Figura 2

Promedios de mediciones Inicial y Final en Abducción de cadera para grupos de Ejercicio y el grupo control

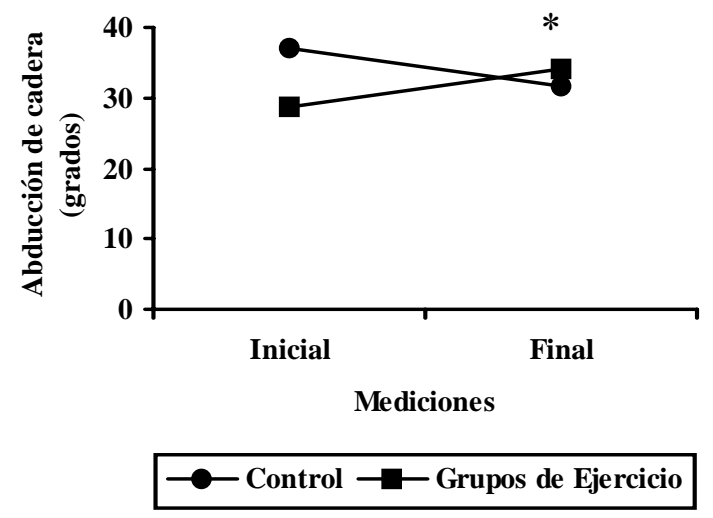

${ }^{*} \mathrm{p}<0.05$ Diferencia significativa entre la medición inicial $\mathrm{y}$ final en los dos grupos que realizaron ejercicio

Figura 3.

Promedios de mediciones Inicial y Final en Aducción de cadera para grupos de Ejercicio y el grupo control

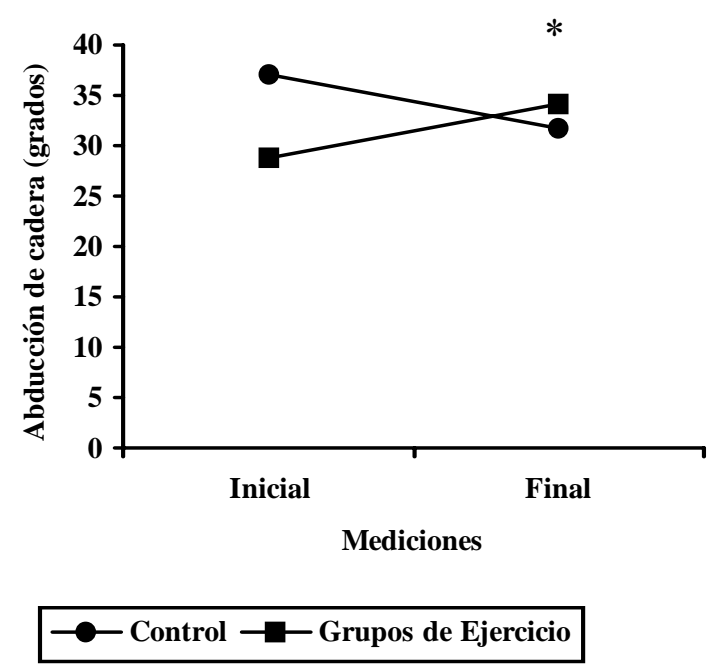

${ }^{*} \mathrm{p}<0.05$ Diferencia significativa entre la medición inicial y final en los dos grupos que realizaron ejercicio
Por último, los efectos simples de las mediciones de rotación externa e interna indicaron que solo el grupo que realizó acondicionamiento físico mostró cambios significativos $(\mathrm{p}<0.05)$ entre el pretest y el postest (ver figuras 4 y 5 ).

Figura 4.

Promedios de mediciones Inicial y Final en Rotación Interna de cadera para grupos de Ejercicio y el grupo control
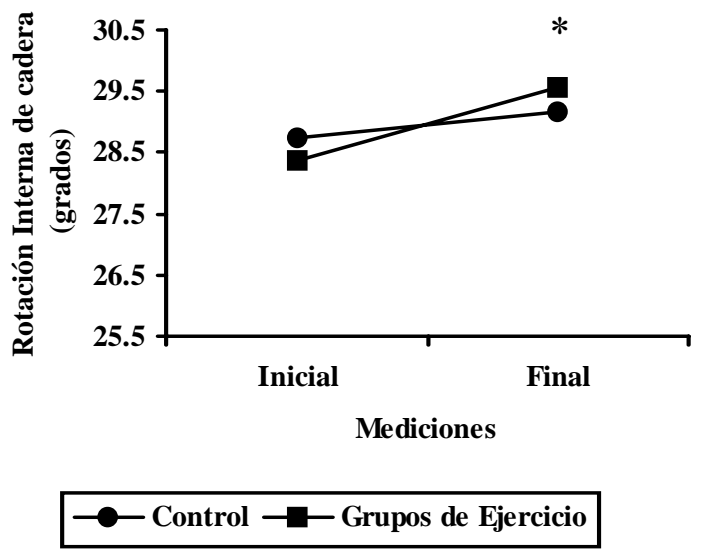

* $\mathrm{p}<0.05$ Diferencia significativa entre la medición inicial y final en los dos grupos que realizaron ejercicio

Figura 5.

Promedios de mediciones Inicial y Final en Rotación Externa de cadera para grupos de Ejercicio y el grupo control

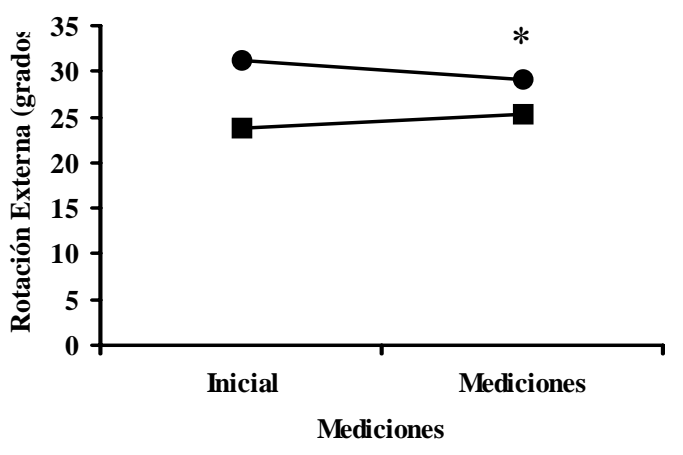

- Control - Grupos de Ejercicio

* $\mathrm{p}<0.05$ Diferencia significativa entre la medición inicial y final en los dos grupos que realizaron ejercicio 


\section{DISCUSIÓN}

El rango de movimiento articular es el resultado de una combinación de factores (Protas, 1998). La estructura de la articulación, por su parte, determina los grados de libertad del movimiento. En el caso particular de la articulación de cadera, ésta permite movimiento en los tres planos: el plano frontal que permite movimientos de abducción y aducción; plano sagital que permite movimientos de flexión y extensión y plano transversal que permite movimientos de rotación (Tórtora y Reynolds, 1998). Para rangos de movilidad articular existen valores promedio para adultos sanos norteamericanos (Heyward, 1998). Cabe decir, que los valores promedio, para los participantes del estudio, tanto antes de los tratamientos como después (ver tabla 1), a excepción de la aducción de cadera, se encuentran por debajo de los valores en sujetos norteamericanos sanos (flexión: $100^{\circ}-120^{\circ}$, Abducción: $40^{\circ}-45^{\circ}$, Aducción: $20^{\circ}-30^{\circ}$, rotación interna: $40^{\circ}-45^{\circ}$, rotación externa: $45^{\circ}-50^{\circ}$ ), estas normas redundan en calificar a los sujetos del presente estudio con presencia de limitaciones en sus movimientos articulares a nivel de cadera, sin embargo los participantes fueron personas adultas mayores sanas y funcionalmente independientes. En este sentido se promueve la determinación de normas nacionales que permitan hacer comparaciones sobre datos de la realidad nacional y así dar validez a la interpretación.

En relación con la pregunta de investigación, ésta fue la siguiente, ¿Se obtendrán resultados distintos al aplicar un programa que combina la hidroquinesia y el acondicionamiento físico y un programa solamente de acondicionamiento físico en la movilidad articular de cadera en población mayor de 60 años?

Desde el punto de vista biomecánico, en teoría, dos aspectos tienden a favorecer la práctica de ejercicios en el agua, para mejorar la flexibilidad. El principio de flotabilidad, el cual lograr reducir la fuerza de compresión articular (Steward y Basmajian, 1986), y así favorecer la amplitud del rango de movimiento. Por otro lado, el medio acuático ofrece continuamente una resistencia al movimiento (Hamill y Knutzen, 1995), la cual el sujeto debe vencer, involucrándose en este sentido ejercicio de fuerza, que fuera del agua no se experimenta.

Sin embargo, los resultados muestran una tendencia distinta, en cuanto a los rangos de movimiento de flexión, abducción y aducción de cadera, en los dos grupos, pues tanto en el que realizó ejercicios en el agua como el que no realizó, se obtuvo una mejoría similar. El grupo control, por su parte, en flexión de cadera mantuvo sus valores iniciales, mientras que en los movimientos de abducción y aducción disminuyeron. Cabe destacar, que el movimiento de flexión de cadera es propio de actividades como el caminar y subir y bajar gradas, que se realizan continuamente, lo que podría explicar que el rango de movimiento en flexión de cadera se mantenga, no así los movimientos de abducción y aducción, lo cual explica la disminución del rango de movilidad articular en el grupo control.

En cuanto a los movimientos de rotación interna y externa, el grupo que realizó el programa de acondicionamiento físico fue el único que presentó cambios, mejorando el rango de movimiento articular.

Los movimientos de rotación interna como externa se ejecutan en mayor o menor grado, según la técnica del caminar, específicamente al virar o cambiar de dirección, estímulo que estuvo presente en las dos sesiones del programa de acondicionamiento físico; sin embargo en el grupo que realizó una sesión de hidroquinesia durante todo el programa, la acción de virarse o cambiar de dirección se ve favorecida por el principio de flotabilidad (Steward y Basmajian, 1986) limitando o reduciendo el estímulo y por ende la mejoría.

En resumen se recomienda tanto la práctica de actividades en medio acuático como fuera de él para mejorar o mantener el rango de movimiento articular global (flexión, abducción, aducción, rotación interna y externa) de la cadera.

Se recomienda para futuras investigaciones medir el componente flexibilidad en unidades angulares, y valorar la acción global de la articulación, es decir, en todos los planos que estructuralmente la articulación lo permita para así determinar con mayor especificidad la influencia de los tratamientos. 


\section{REFERENCIAS}

American College of Sports Medicine (2000). ACSM's Guidelines for Exercise Testing and Prescription $\left(6^{\text {th }}\right.$ Ed.) USA: Williams \& Wilkins.

Aniansson, A y Gustafsson, E. (1981) Physical training in old men with special reference to quadriceps muscle strength and morphology. Clinical Physiology 1, 87-98.

Baylor; A.M.; Spirduso, W.W. (1988) Systematic aerobic exercise and components of reaction time in older women. Journal of Gerontology. 43, 121-126.

Carter, D.R., Fyhrie, D.P. \& Whalen, R.T. (1987). Trabecular bone density and loading history: regulation of connective tissue biology by mechanical energy. Journal of Biomechanics, 20, 785-794.

Clark, B. (1986) Exercise Programs for Older Adults. JOPERD. 12(4), 63-65.

Clarkson, P.M.; Kroll, W. (1978) Practice effects on fractionated response time related to age and activity level. Journal of Motor Behavior. 10, 275-286.

Fiatarone, M., Marcks, E., Ryan, N., Meredith, C., Evans, W. (1990). High-intensity strength training in nongenarians: Effect on skeletal muscle. JAMA. 263:3029-3034.

Hamill, J. y Knutzen, K.M. (1995). Biomechanical Basis of Human Movement. Williams \& Wilkins.

Harris, C., M. Debeliso, T.A. Gigson, K. Fitzgerald (2001). Effect of resistance Trainnig Intensity on strength gain in the older adults. Medicine \& Science in Sports \& Exercise. 31(5) suplemento.

Heyward, V. (1998). Advanced Fitness Assessment Exercise Prescription. 3era. Edición. (pp.205-211). Human Kinetics.

Israel, S. (1992). Age-related Changes in Strength and Special Groups. En Komi, P.V. (Ed.). Strength and Power in Sport (pp. 319-328). Oxford, Inglaterra: Blackwell Scientific Publications, Vol. III

Klein, D.A. (2001). Does flexibility training improve physical function in assisted-living older adults? Suplemento Medicine and Science in Sports and Exercise 33 (5): S344.

Lan, C., Lai, J.S., Chen, S.Y., Wong, M.K. (1998) 12-month Tai Ci training in the elderly: its effect on health fitness. Medicine and Science in Sports and Exercise 30(3):345351.

Mcginnis, J.M. (1992) The Public Health burden of sedentary lifestyle. Medicine and Science in Sports and Exercise, 196-200.

Meredith, C.N., Frontera, W.R., Fischer, E.C. (1989). Peripheral effects of training in young and old Subjects. J. Applied Physiology. 66:2844-2849

Miller, R. (1994). The biology of aging and longevity. Principles of Geriatric Medicine and Gerontology. Mac Graw-Hill, Inc.

Mobily, K.E., Mobily, P.R., Lane, B.K. y Semerjian, T.Z. (1998) Using progressive resistance training as an intervention with older adults. Therapeutic recreation Journal 32(1):42-53

Nieman, D. (2000). ACSM'S Exercise Soothes Arthritis. Health \& Fitness Journal. 4 (3), 20-27.
Ortiz, E. (1998). Ejercicio, envejecimiento y Bienestar. Kinesis, 2,16-22.

Protas, E. (1998). Flexibility and range of Motion. ACSM'S Resource Manual for Guidelines for Exercise Testing and Prescription. 3era. Edición. Capítulo 44. Williams y Wilkins.

Rigal, R. (1987). Motricidad Humana. Madrid. Editorial Pila Teleña S.A.

Rikli; R.E., Busch, S. (1986) Motor perfomance of women as a function of age and physical activitylevel. Journal of Gerontology. 41, 645-649.

Schroeder, J.M., Nau, K.L., Osness, W.H., Pottieger, J.A. (1998) A comparison of life satisfaction, functional ability, physical characteristics and activity level among older adults in various living settings. Journal of aging and physical activity, 6(4):340-349.

Sergent, E.H. (1995) The effect of exercise on stress and functional abilities in community dwelling elderly. Tesis de Maestría sin publicar, Purdue University.

Shepard, R.J. (1994). Physiological basis of training in the elderly. Science and Sports 9(4):189-196

Solano, L.C., Vargas, O. (1999) Efecto de un Programa de Hidroterapia en las Condiciones Osteomusculares y en la Autoestima en un Grupo de Mujeres Adultas Mayores. Revista Gerontológico. CENDEISS-CCSS. (1), 33-48.

Spirduso, W. y Clifford, P. (1978) Replication of age and physical activity effects on reaction and movement time. Journal of Gerontology. 33, 26-30.

Stewart, J. y Basmajian, J.(1986). Ejercicios en el agua. En Basmajian, J. (Ed.). Terapéutica por el Ejercicio_(3 ed.). Buenos Aires, Argentina: Edit. Médica Panamericana.

Tórtora, G. y Reynolds, S. (1998). Principios de Anatomía y Fisiología ( $7^{\text {ma }}$ Ed.). Madrid. Harcourt Brace.

Williams, E. (1999). Exercise training guidelines for elderly. Medicine \& Science in Sports \& Exercise. Vol.32 (1).

Willmore J. y Costill D. (1999). Changes in Strength with Aging. Physiology of Sports and Exercise. 2nda edición. Human Kinetics. U.S.A. 